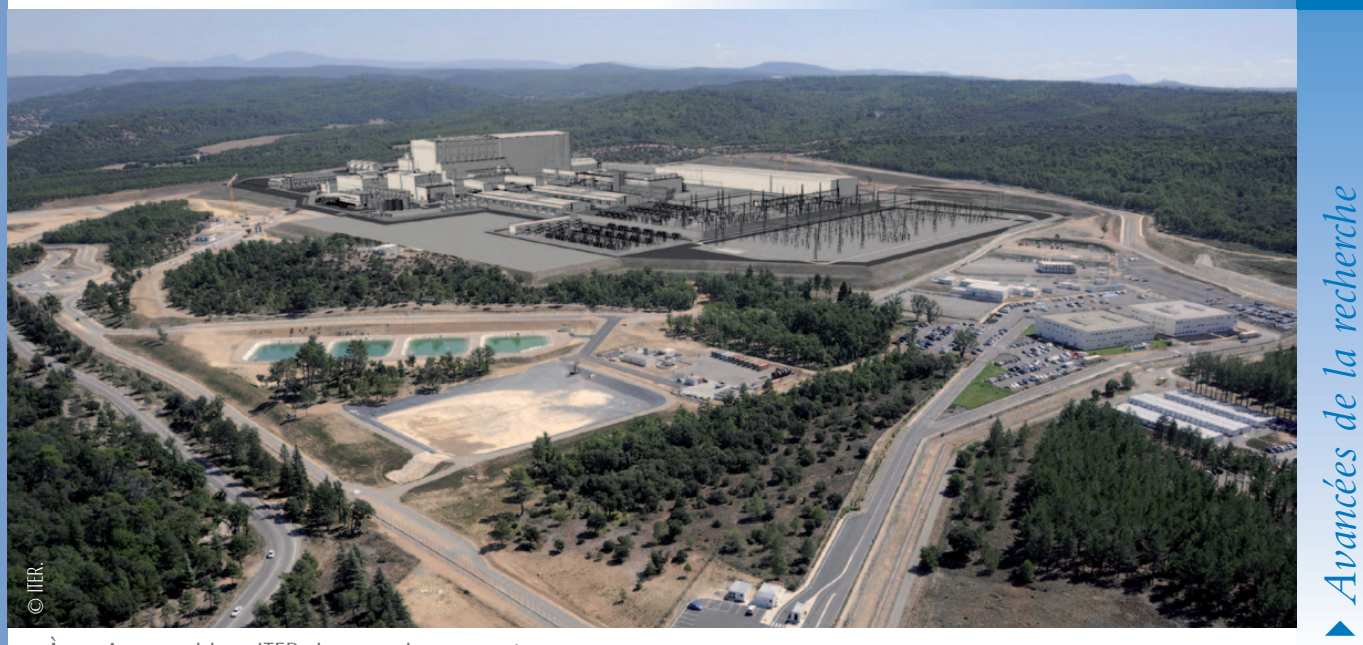

À quoi ressemblera ITER dans quelques années.

\title{
ITER et les recherches sur la fusion par confinement magnétique
}

Jean Jacquinot (jean.jacquinot@cea.fr)

Conseiller scientifique auprès de l’Administrateur général du CEA, CEA/Saclay, 91191 Gif-sur-Yvette Cedex

La fusion nucléaire contrôlée

\section{présente des avantages}

de premier ordre en matière de sûreté nucléaire, mais sa disponibilité nécessite

I'aboutissement d'un important programme de recherches en amont de la phase industrielle.

ITER, « le chemin » en latin, est la clé de voûte du programme de recherches sur la fusion

par confinement magnétique.

Sa construction est en cours, sous la direction d'une vaste collaboration internationale dont le siège est à Cadarache, en Provence. L'état et les points essentiels de cette recherche sont précisés, ainsi que ses nombreuses opportunités pour la science française.

\section{Motivations}

Le choix d'un bouquet énergétique adapté à la société du $21^{\mathrm{e}}$ siècle est plus que jamais à l'ordre du jour. Alors que plus d'un milliard d'habitants vivent sans électricité, la population mondiale ne cesse de croître et, avec elle, la demande en énergie. L'urgence est particulièrement forte dans les pays en voie de développement. Malgré un effort important dans le domaine des énergies renouvelables, cette demande supplémentaire est, à l'échelle mondiale, satisfaite dans une très large mesure par un recours toujours plus grand aux combustibles hydrocarbonés et par un retour massif à l'utilisation du charbon. Les climatologues, de leur côté, lancent des cris d'alarme sur les effets probables du réchauffement climatique. C'est l'un des défis majeurs de ce siècle que de concilier la croissance inévitable de la consommation énergétique mondiale avec l'inéluctable épuisement des ressources fossiles et le respect de notre environnement (en 2010, 80,4\% de l'énergie consommée provenait de la combustion des énergies fossiles). Pour y parvenir, la seule approche envisageable est d'économiser davantage l'énergie et d'accentuer l'effort de recherche et de développement pour un usage, optimisé et combiné, des ressources renouvelables et des ressources nucléaires disponibles et sûres. En l'absence de stockage efficace de l'énergie intermittente en provenance des sources renouvelables, il est en effet improbable que ces dernières puissent à elles seules remplacer les sources génératrices de gaz à effet de serre. Le recours à l'énergie nucléaire pour la fourniture de l'électricité de base des grandes concentrations industrielles paraît inévitable, mais les évènements tragiques qui se sont produits en mars 2011 au Japon renforcent chaque jour davantage les exigences de la sûreté face aux risques nucléaires.

On sait que l'énergie tirée de la fusion des isotopes de l'hydrogène, si elle pouvait devenir disponible industriellement, éviterait l'essentiel des risques associés à l'énergie de fission : pas de risque d'emballement (pas de réactions en chaîne et pas plus de quelques grammes de combustible dans la machine sous forme de plasma ténu), faible chaleur résiduelle décroissant très vite dès l'arrêt du réacteur, pas de matériaux fissiles permettant la confection d'un détonateur nucléaire (donc pas de risques de prolifération), pas de déchets radioactifs à extrêmement longue durée.

Depuis la fin des années 60, deux voies ont été développées pour la fusion thermonucléaire contrôlée : le confinement inertiel [1] et le confinement magnétique. C'est dans le cadre de cette seconde voie qu'a été développée la filière des "tokamaks" (voir encadré p. 22), d'abord expérimentée avec succès en URSS sur une idée d'Andrë Sakharov. À partir de 1970, des installations de plus en plus performantes ont été construites et exploitées de par le monde. ITER est la prochaine étape. 


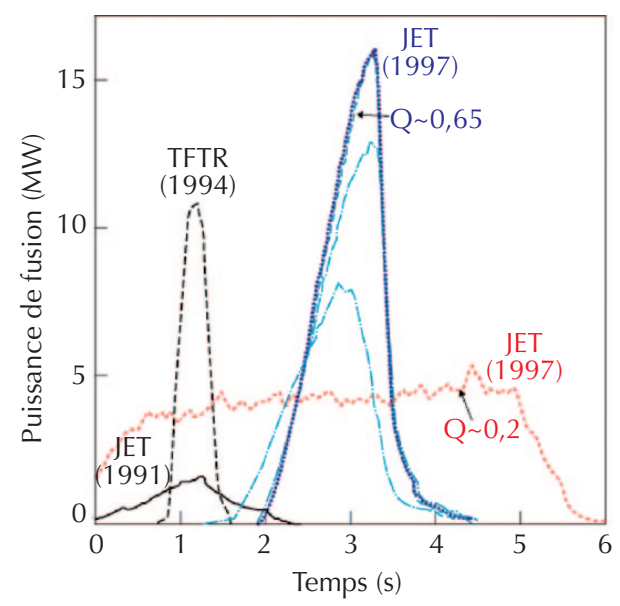

1. Évolution temporelle de la puissance de fusion obtenue dans les deux expériences ayant fonctionné avec un mélange deutérium-tritium : le TFTR de l'université de Princeton et l'expérience européenne JET. Q est le gain défini dans le texte. ITER est dimensionné par rapport au gain $Q \sim 0$,2, qui permet de fonctionner en régime stationnaire. Sa taille doit permettre d'obtenir $Q=10$ dans ce régime.

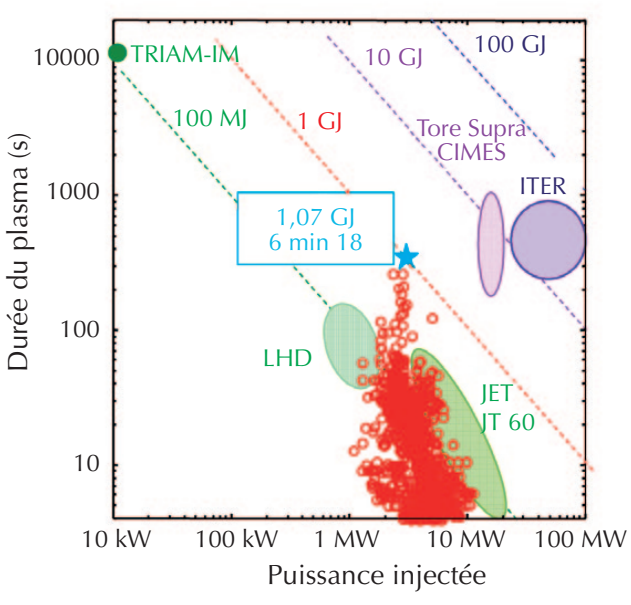

2. Progrès dans la durée des décharges longues dans les tokamaks, en fonction de la puissance injectée (d'après [4]). Les ronds rouges correspondent aux résultats expérimentaux de Tore Supra. Récemment, le stellarator japonais LHD a dépassé la marque du GJ, avec une puissance de chauffage de l'ordre du MW. l'objectif d'ITER est de fonctionner par impulsions répétitives de $400 \mathrm{~s}$.

\section{\〉 \\ La fusion nucléaire contrôlée : une longue marche [2]}

Depuis Rutherford dans les années 30, on sait faire la fusion des isotopes de l'hydrogène. La fusion entre deux noyaux de deutérium fut même la toute première découverte faite avec un accélérateur de particules. Mais la réaction la plus favorable est la fusion entre un noyau de deutérium (noté $\mathrm{D}$ ou ${ }^{2} \mathrm{H}$ ) et un noyau de tritium (noté $\mathrm{T}$ ou ${ }^{3} \mathrm{H}$ ), dite réaction $\mathrm{D} / \mathrm{T}$, qui donne un noyau d'hélium et un neutron, et qui a la plus grande section efficace, avec un maximum à une énergie relativement basse, de $100 \mathrm{keV}$ :

$$
\begin{aligned}
& { }^{2} \mathrm{H}+{ }^{3} \mathrm{H} \rightarrow{ }^{4} \mathrm{He}(3,56 \mathrm{MeV}) \\
& +{ }^{1} \mathrm{n}(14,03 \mathrm{MeV})
\end{aligned}
$$

Dans l'exposé de ses résultats, Rutherford avait tout de suite remarqué que, bien que le processus soit fortement exothermique, la section efficace était insuffisante pour obtenir un gain net d'énergie par une interaction faisceau-cible. John Lawson établira, vingt-cinq ans plus tard, qu'un milieu thermiquement isolé permettrait de réaliser une production nette d'énergie à condition que le produit entre la densité des ions (n) et le temps de confinement de l'énergie $(\dagger) \quad\left(\tau_{E}\right)$ soit supérieur à une certaine valeur, et que la température $T$ soit suffisamment élevée. Pour la réaction $\mathrm{D} / \mathrm{T}$, le critère de Lawson s'écrit n. $\tau_{\mathrm{E}} \geq 1,5 \times 10^{20} \mathrm{~m}^{-3}$.s : typiquement, il faut maintenir une densité de $10^{20}$ ions par $\mathrm{m}^{3}$ à une température de $10 \mathrm{keV}\left(\sim 10^{8} \mathrm{~K}\right)$, avec un temps de confinement de l'énergie supérieur à 3 secondes.
Dans le cas de la fusion par confinement magnétique, l'objectif est que le triple produit $n . \tau_{\mathrm{E}} \cdot T$ atteigne la valeur minimale de $310^{21} \mathrm{~m}^{-3}$. keV.s.

Quatre-vingts ans après Rutherford, on n'a toujours pas réussi à réaliser un réacteur à bilan positif, malgré un effort de recherche très substantiel. C'est qu'il a fallu d'abord établir les fondations de la physique des plasmas avec ses très complexes effets collectifs, qui se sont révélés bien plus difficiles à maîtriser que prévu. Aujourd'hui, la physique des plasmas est une science solidement établie, avec de nombreuses applications dans le domaine industriel.

Dans les expériences de fusion par confinement magnétique en géométrie torique (tokamaks et stellarators $(†)$ ), les instabilités macroscopiques du plasma, même dans le domaine non linéaire, sont maintenant bien maîtrisées et les performances de ces machines, mesurées par le triple produit n. $\tau_{\mathrm{E}} . T$ et par la durée de décharge, ont toutes les deux fait des progrès d'un facteur d'environ 10 000. Pendant de courtes durées, le JET $(\dagger)$ a ainsi réalisé plus de $16 \mathrm{MW}$ de fusion en utilisant un mélange D/T ([3] et fig. 1), et le Tore Supra à Cadarache a démontré un fonctionnement stable pendant une durée supérieure à 5 minutes [4]. Une étape importante a aussi été franchie en technologie, avec l'utilisation de supraconducteurs à haut champ critique et de surfaces refroidies en continu face au plasma, ces dernières démontrant une bonne tenue avec une charge thermique au niveau requis de $10 \mathrm{MW} / \mathrm{m}^{2}$. On a atteint et même dépassé, dans plusieurs grands tokamaks, les températures et les densités requises, mais il reste encore à améliorer la valeur $\mathrm{du}$ temps de confinement $\tau_{\mathrm{E}}$. Celui-ci croît rapidement avec la taille du plasma et la valeur du champ magnétique. Les figures 1, 2 et 3 illustrent les performances atteintes dans les tokamaks. ITER est conçu pour augmenter $\tau_{\mathrm{E}}$ jusqu'à une valeur de 2 à 5 secondes (facteur 3 par rapport à la meilleure performance du JET), de façon à obtenir un gain $\mathrm{Q}$ (rapport de la puissance dégagée par la réaction de fusion à la puissance de chauffage) de 10 .

Il ne fait maintenant plus de doute que le principe de confinement en géométrie toroïdale doit permettre, si la taille est suffisante et sous réserve d'une technologie appropriée, le gain d'énergie nécessaire au fonctionnement d'un réacteur. Il reste à préciser cette taille dans les conditions réelles de fonctionnement, c'est-à-dire en "brûlant " un mélange D/T. Le déploiement de tokamaks dans tous les pays industrialisés a permis d'établir une loi d'échelle pour établir ce dimensionnement.

\section{Notion de taille critique, lois d'échelle, dimensionnement d'ITER et calculs ab initio}

Dans un premier temps, les instabilités magnétohydrodynamiques qui se développent à grande échelle ( rayon du plasma) ont été maîtrisées en évitant l'espace opérationnel où elles peuvent se développer. La qualité du confinement du plasma est dès lors entièrement définie par 


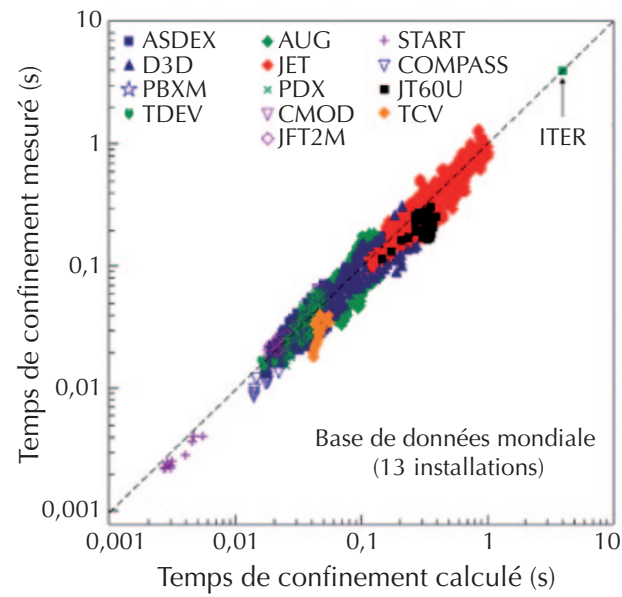

3. Loi d'échelle sur le confinement : comparaison calculs/expériences. Représentation en coordonnées logarithmiques de la base de données mondiale des tokamaks de forme semblable, mais de dimensions et de champs magnétiques très différents. Pour chaque machine, on a porté en ordonnée le temps de confinement de l'énergie mesuré expérimentalement, et en abscisse le même temps calculé suivant la loi d'échelle: $B \tau_{\mathrm{F}}=\left(\rho^{*}\right)^{-2,7} \beta^{-0,9}\left(\boldsymbol{v}^{*}\right)^{-0,01}$, proche de la forme théorique électrostatique (équation 2). Tous les points s'alignent sur une droite de pente 1.

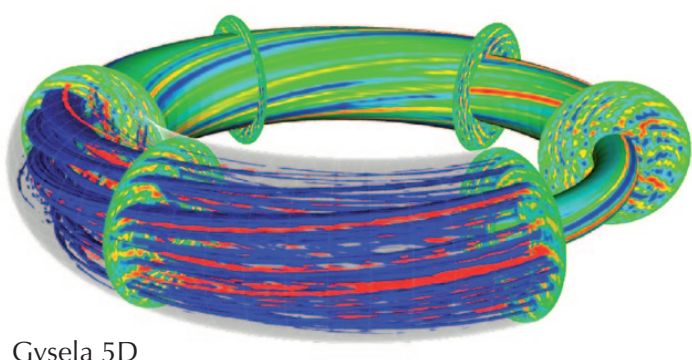

Gysela 5D la turbulence générée par des instabilités microscopiques (échelle : $\sim$ rayon de Larmor $(\dagger)$ des ions dans le champ poloïdal), qui se traduisent par une diffusion de la chaleur depuis le centre chaud jusqu'au bord. Le taux de diffusion correspondant détermine la valeur du temps de confinement $\tau_{\mathrm{E}}$. Il est donc essentiel d'établir les lois d'échelle qui lient $\tau_{\mathrm{E}}$ aux différents paramètres du problème : dimensions de la configuration, forme, valeur du champ magnétique et caractéristiques du plasma. En raison du caractère diffusif d'un tel processus, on s'attend à ce que $\tau_{\mathrm{E}}$ augmente rapidement avec la taille du plasma et qu'il existe donc une dimension minimum pour qu'un réacteur puisse obtenir un gain en énergie.

La détermination des lois d'échelle du confinement revêt donc une importance primordiale. Elle a pu être réalisée en utilisant le principe de similitude, déjà employé avec grand succès pour les problèmes d'hydrodynamique, par exemple pour dimensionner les avions en soufflerie. La collaboration entre les différentes équipes en charge des tokamaks a permis de rassembler une base de données des résultats expérimentaux en fonction des paramètres sans dimension de la magnétohydrodynamique. L'étude ([5], p. 72) a montré que le confinement peut s'exprimer en fonction de trois paramètres sans dimension : le rayon de Larmor $(\dagger)$ normalisé au petit rayon moyen du plasma $\left(\rho^{\star}\right)$, la pression du plasma normalisée à la pression magnétique $\left(\beta^{\star}\right)$ et la fréquence de collision coulombienne normalisée au temps nécessaire à une particule pour faire le tour du tore $\left(v^{\star}\right)$. Une loi unique peut représenter les résultats de tous les tokamaks de forme comparable. L'expression théorique, déduite avec la contrainte que la turbulence est de nature électrostatique, s'écrit [6], B étant la valeur du champ magnétique :

$$
\text { B. } \tau_{E} \propto\left(\rho^{\star}\right)^{-3} \cdot \beta^{0} \cdot\left(v^{\star}\right)^{0}
$$

Cette loi met bien en évidence l'importance de la taille du plasma (qui intervient au cube par $\left.\left(\rho^{\star}\right)^{-3}\right)$ et de la valeur du champ magnétique (qui intervient au carré par le rayon de Larmor, mv/eB) sur la valeur du temps de confinement.

Par ailleurs, la loi d'échelle déduite des résultats expérimentaux (fig. 3) est très proche de l'expression théorique (2). Elle a servi à dimensionner le projet ITER, de façon à obtenir un gain $Q$ de 10 en puissance de fusion par rapport à la puissance injectée.

Malgré les succès des lois d'échelle obtenues par la méthode des similitudes, une description précise du transport par le calcul $a b$ initio est très souhaitable. Cependant, un tel calcul reste, de par son extrême complexité, un grand défi pour la discipline. Des progrès importants viennent d'être obtenus grâce à la simulation numérique et à la puissance des ordinateurs modernes. Il est maintenant possible de simuler le cœur du plasma d'un tokamak [6], en prenant en compte sa géométrie tridimensionnelle et l'évolution de la fonction de distribution des ions. Le calcul confirme la dépendance en $\rho^{\star}$ et montre que le transport de la chaleur se fait par une multitude de cellules de convection tourbillonnantes (fig. 4). La théorie et l'expérience indiquent que le transport peut être réduit localement en modifiant le profil du champ magnétique poloïdal de façon à cisailler les lignes de champ, ce qui limite l'extension radiale de ces structures. Cette voie très prometteuse, souvent appelée « tokamak avancé ", fait l'objet de recherches dans de nombreuses installations de taille moyenne. Le dimensionnement d'ITER a été déterminé sans tenir compte de ce bonus. Cependant, les trois systèmes de chauffage du plasma et de génération de courant, totalisant une puissance de $73 \mathrm{MW}$, sont conçus pour permettre d'explorer cette possibilité.

$\gg>$

Les paramètres d'ITER ont ainsi été définis aux valeurs suivantes :

\begin{tabular}{|l|l|}
\hline Grand rayon du tore & $6,2 \mathrm{~m}$ \\
\hline Petit rayon (plan équatorial) & $2 \mathrm{~m}$ \\
\hline Champ toroïdal B & $5,3 \mathrm{~T}$ \\
\hline Volume du plasma & $678 \mathrm{~m}^{3}$ \\
\hline Courant circulant dans le plasma & $15 \mathrm{MA}$ \\
\hline Puissance de chauffage auxiliaire & $73 \mathrm{MW}$ \\
\hline Puissance générée et gain & $500 \mathrm{MW} ; \mathrm{Q}=10$ \\
\hline Durée de maintien du plasma & $400 \mathrm{~s}$ \\
\hline
\end{tabular}




\section{Confinement magnétique et tokamak}

Dans une géométrie cylindrique infinie, les particules chargées s'enroulent autour des lignes de champ magnétique, et restent confinées à la condition que les collisions soient rares. Le confinement magnétique isole ainsi de la paroi, qui pourrait le refroidir, un plasma chaud et ténu, donc peu collisionnel. II reste à éviter les pertes aux extrémités en refermant le cylindre sur lui-même, ce qui forme un tore. Cependant, en courbant le champ magnétique on induit une dérive verticale des particules chargées vers la paroi. Dans un tokamak, ces dérives sont compensées par l'effet du champ créé par le courant dans le plasma lui-même, et par des bobines de compensation. Un plasma contenant $10^{20}$ ions $/ \mathrm{m}^{3}$ est le siège $\mathrm{d}^{\prime}$ abondantes réactions nucléaires de fusion lorsque la température dépasse $10^{8} \mathrm{~K}(10 \mathrm{keV})$. Un bilan positif d'énergie est attendu, pourvu que ces conditions puissent se maintenir suffisamment longtemps (critère de Lawson).

Un anneau de plasma placé dans un champ magnétique torö̈dal créé par un solénoïde replié est le plus simple des systèmes fermés. Dans un tokamak, le plasma est parcouru par un fort courant toroïdal obtenu par induction, l'anneau étant le secondaire à un tour d'un transformateur. Ce courant crée un champ magnétique polö̈dal qui contribue à la formation de lignes de champ enroulées en hélice sur des tores emboîtés. Ce sont des «surfaces magnétiques », contenant aussi les lignes de courant, et sur lesquelles la pression de plasma est constante (fig. A). Le plasma participe ainsi à son propre confinement. Sa frontière est une surface magnétique.

Dans une telle configuration, les dérives sont compensées et les particules confinées suivent les deux types de trajectoires représentées sur la figure B. Le champ magnétique, dont la grandeur décroît lorsqu'augmente la distance à l'axe, est à l'origine d'un effet miroir qui maintient une partie des particules dans les régions à champ faible, tandis que d'autres (circulantes) ont accès aux zones à champ fort tout en restant confinées.

Une autre façon d'envisager le confinement consiste à considérer le plasma comme un fluide immergé dans le champ magnétique et à raisonner en termes de densité d'énergie. À l'équilibre, la somme de la pression et de la densité d'énergie magnétique est une constante égale à la densité d'énergie magnétique hors de l'anneau de plasma. Il est difficile de faire durer un tel équilibre perturbé par de multiples causes : fuites, instabilités... qu'il convient d'étudier et de maîtriser. C'est tout l'objet de la recherche sur les plasmas de fusion par confinement magnétique.

Jean-Louis Bobin Professeur émérite, Université Pierre et Marie Curie (Paris 6)

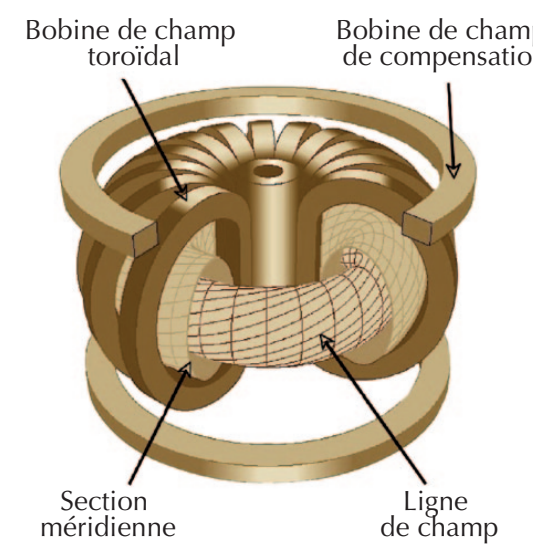

A. Schéma de principe d'un tokamak. Le solénoïde replié est constitué de bobinages disposés en couronne autour de l'axe du tore. La forme en D est caractéristique des grands tokamaks. Les lignes de champ s'enroulent sur des surfaces magnétiques, tores emboîtés de section méridienne allongée dans le sens de la hauteur. Le champ de compensation, vertical, évite à l'anneau de plasma de se dilater.

\section{\> \\ ITER : conception, technologie, validation, organisation}

Entre 1992 et 2001, la conception détaillée et l'ingénierie d'ITER ont été confiées à une équipe internationale appartenant aux quatre partenaires d'origine (Europe, Japon, Russie et USA jusqu'en 1997), qui a remis en 2001 un rapport détaillé aux partenaires. La période qui suivit fut consacrée à une consolidation technique et à la réalisation de prototypes des éléments clés du projet. Le public se souviendra aussi des négociations pour le choix du site et de la compétition intense entre quatre sites candidats (Aomori au Japon, Cadarache en France, Clarington au Canada et Vandellos en Espagne). On sait moins que les scientifiques des pays partenaires furent conviés à une revue très détaillée des éléments scientifiques et techniques du projet. Cette revue, qui a continué jusqu'à une époque très récente, a conduit à un certain nombre d'améliorations et de changements. Ces changements, combinés au renchérissement des matériaux et à la multiplication des interfaces avec un nombre accru de partenaires qui passeront à sept en 2006, ont certainement beaucoup contribué à une augmentation du coût de la machine et des délais pour sa réalisation.

Ce processus presque continu de revue scientifique a conduit à une adhésion très forte des communautés compétentes dans le sujet. À titre d'exemple, la communauté scientifique américaine fut rassemblée en 2002 à Snowmass pour débattre de l'étape "burning plasma", indispensable à l'avenir du projet. Cela conduisit la National Academy à une recommandation extrêmement forte [7] pour que les États-Unis redeviennent partenaires d'ITER, ce qu'ils firent en 2004. L'Europe, sous la direction de la Commission Européenne suivit une démarche semblable. En France, on notera le rapport scientifique et technique de l'Académie des sciences [5] qui étudia en détails l'état de la science et de la technologie, ainsi que les verrous à la fois pour la fusion par confinement magnétique (ITER) ou inertiel (LMJ).

Depuis le 24 octobre 2007, les sept grands partenaires (Chine, Corée (du Sud), Europe (Suisse incluse), Japon, Inde, Russie et États-Unis) ont formellement ratifié, sous l'égide de l'AIEA, l'accord qui institue l'organisation internationale ITER dont le siège est à Cadarache. L'accord stipule la fourniture en nature par chaque 


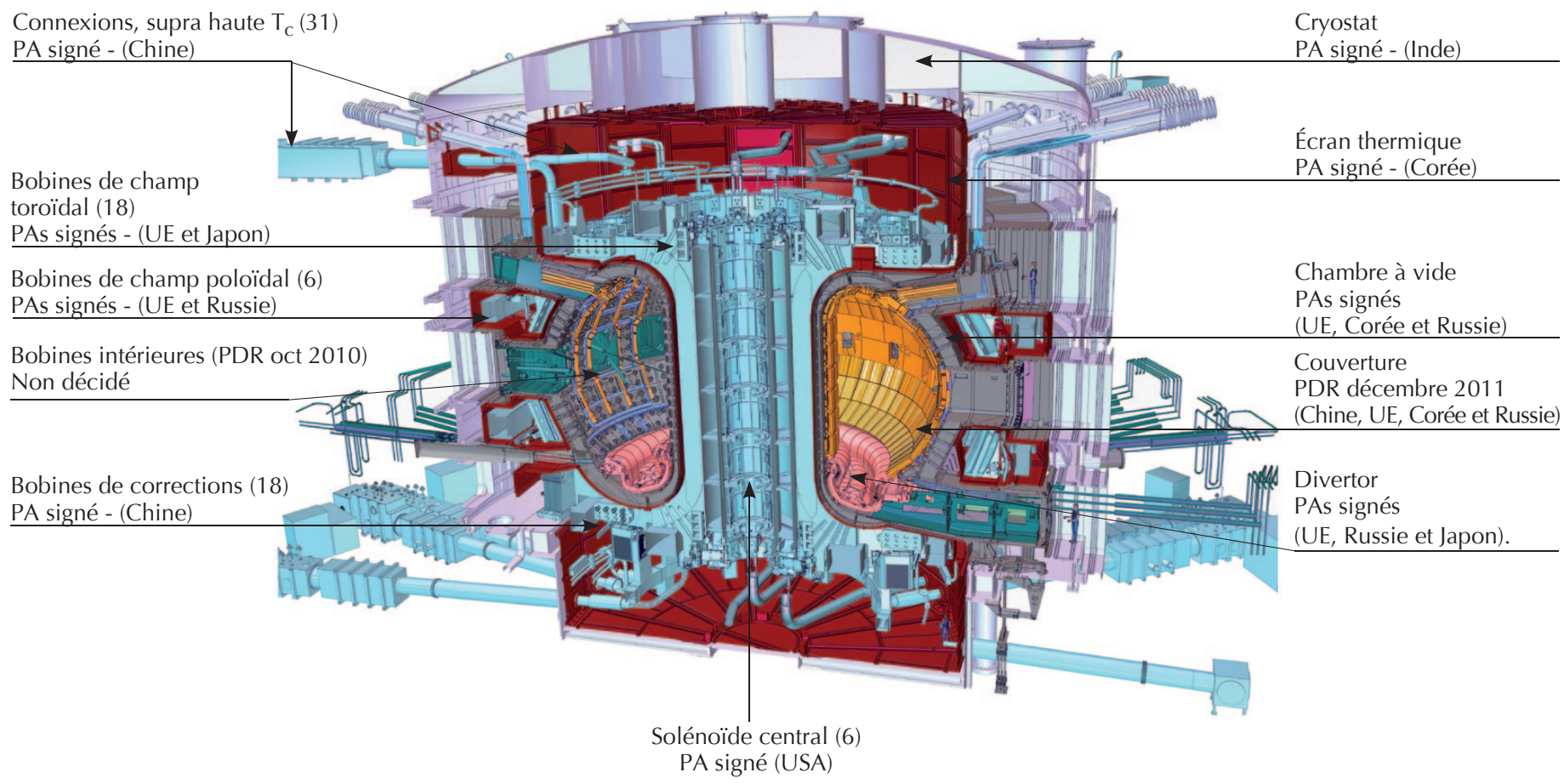

5. Éclaté d'ITER, permettant de distinguer les principaux éléments de la machine et l'état d'avancement de la construction en septembre 2012. De l'extérieur vers l'intérieur : le cryostat qui enferme l'ensemble, l'écran thermique (en rouge), les bobines de champ toroïdal (en bleu), la chambre à vide (en gris), les modules de couvertures (en jaune), les bobines intérieures (en orange), le divertor (en rose). Enfin, la colonne centrale est constituée d’un empilement de six galettes formant le solénoïde central. PDR = Preliminary Design Review; PA = Procurement Arrangement (dernière étape avant le lancement de la construction).

partenaire d'une partie de la machine. Chaque partie a été évaluée, non pas en valeur monétaire mais en fraction du travail global à fournir. C'est ainsi que l'Europe, qui bénéficie du choix du site, doit fournir $46 \%$ du total de la construction, et chacun des six autres partenaires 9\%. Ces fournitures en nature seront réalisées par les agences domestiques de chaque partenaire, sous le contrôle de l'organisation internationale qui définit les spécifications. En marge de ces accords, l'Europe et le Japon se sont engagés sur "l'approche élargie ", pour une valeur estimée à $10 \%$ du coût d'ITER, à partager également entre les deux partenaires, et qui comprend trois éléments réalisés au Japon : l'EVEDA d'IFMIF ("Engineering Validation Engineering Design Activities" de 1'“International Fusion Irradiating Facility"), une participation au tokamak japonais JT60SA et un "International Fusion Energy Research Center”, doté d'importants moyens de calcul. Ce centre de calcul est désormais en fonctionnement.
La figure 5 illustre les principaux composants de la machine, la distribution des fournitures qui doivent être délivrées par les partenaires et leur état d'avancement. La quasi-totalité de ces fournitures est engagée (fig. 5). La construction à grande échelle a démarré pour les bâtiments, la chambre à vide et les bobines magnétiques. L'effort est particulièrement impressionnant dans le domaine des supraconducteurs, qui constituent environ 30\% de l'effort global : en septembre 2011, 450 tonnes de brins supraconducteurs en $\mathrm{Nb}_{3} \mathrm{Sn}(\sim 81000 \mathrm{~km})$ avaient déjà été produites, soit $70 \%$ de la quantité nécessaire. Cette production répartie à travers le monde (UE, Russie, Japon, Chine et Corée) constitue une augmentation de deux ordres de grandeur sur la capacité mondiale de production de l'industrie avant ce développement. Côté français, on notera que les engagements essentiels concernant le site de Cadarache ont été tenus (itinéraire pour convoi exceptionnel, école internationale, viabilisation du site). Le site a été remis par la France à l'organisation internationale. La construction du bâtiment nécessaire à la réalisation des grandes bobines de champ poloïdal et les fondations antisismiques du bâtiment principal sont terminées (fig. 6). De 2008 à septembre 2012, plus de $1000 \mathrm{M} €$ de contrats ont été attribués à des entreprises françaises, dont plus de $60 \%$ dans la région PACA ; on notera que le retour économique en région a déjà largement dépassé le niveau du financement volontaire des communautés locales, qui est pourtant considérable (467 M€).

Une étape majeure de l'histoire du programme a été franchie le 10 novembre 2012 quand le Journal officiel a publié le décret, signé par le Premier ministre sur recommandation de l'Autorité de sûreté nucléaire, autorisant l'organisation internationale ITER à créer l'installation nucléaire de base sur la commune de Saint-Paul-lez-Durance, permettant ainsi la poursuite des travaux de construction. 

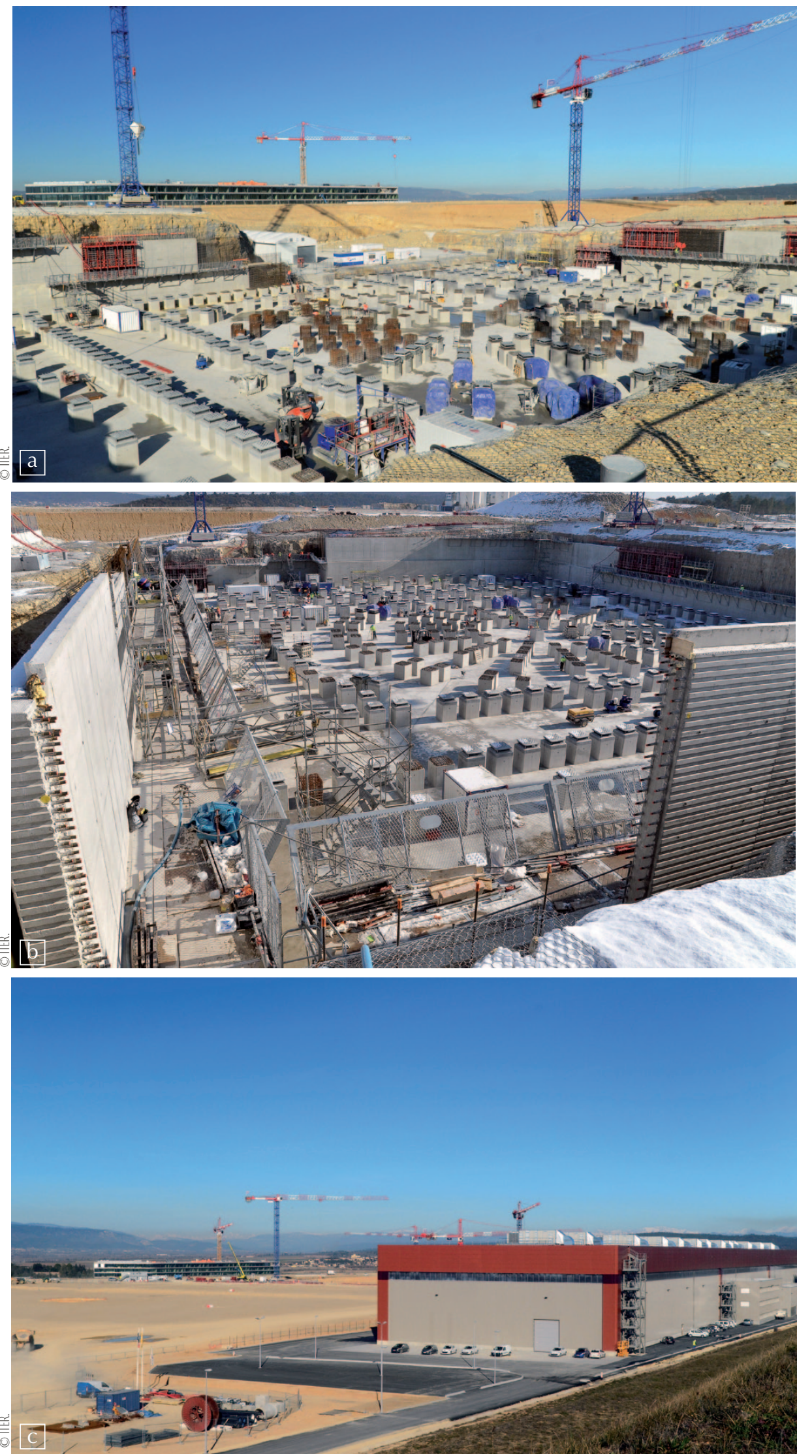

6. Quatre photos prises sur le site d'ITER en 2012. Les vues (a) et (b) montrent les fondations du hall tore équipées du système d'isolation sismique. Les vues (c) et (d) montrent le grand hall qui servira à construire sur place les grandes bobines de champ poloïdal. Le bâtiment qui abrite le siège de l'organisation internationale est visible en arrière-plan sur les vues (a) et (c) ; il a été inauguré le 17 janvier 2013. $\gg>$

\section{Défis et opportunités pour la science française}

Les défis associés à la réussite du projet ITER sont à la fois de nature scientifique et technique.

Sur le plan scientifique, l'un des objectifs majeurs est la validation des lois d'échelle dans les conditions d'un réacteur brûlant un mélange $\mathrm{D} / \mathrm{T}$. Mais on attend bien plus qu'une confirmation de l'équation (2). Il faut explorer un domaine tout à fait nouveau, où l'énergie du plasma est pour l'essentiel $(\sim 2 / 3)$ fournie in situ par la population de particules alpha (noyaux d'hélium $\mathrm{He}^{2+}$ ) résultant des réactions de fusion (cf. équation (1)). La distribution des vitesses de ces particules alpha se trouve en grande partie au-delà de la vitesse d'Alfvén ( $\dagger$ ), et peut être à l'origine d'instabilités magnétohydrodynamiques spécifiques. On s'attend à une situation marginalement stable dans le régime nominal $(Q=10)$; mais, en régime avancé avec un profil de courant optimisé, le développement non linéaire de ces instabilités pourrait bien provoquer des surprises sur l'équilibre final. Les équipes scientifiques françaises, parmi les meilleures au monde dans la théorie, la modélisation et la simulation des tokamaks, devraient jouer un tout premier rôle dans ce domaine.

Les régimes de fonctionnement du tokamak sont nombreux, et devront être documentés avec un ensemble de diagnostics qui devrait atteindre la centaine en phase de pleine exploitation. Dans ce domaine, également, les équipes françaises se préparent à une contribution majeure, entre autres dans l'optique infrarouge, les micro-ondes, les mesures magnétiques, etc. Une "Fédération nationale de recherche sur la fusion par confinement magnétique - ITER » a été créée et prépare activement la phase d'exploitation d'ITER. Elle procède à des tests de validation sur des expériences existantes. Cette fédération regroupe actuellement des scientifiques répartis dans 25 laboratoires, appartenant au CEA, au CNRS, à l'INRIA et aux universités. De nombreux contrats ANR ont été obtenus dans ce cadre. Ce dispositif est complété par la création du Laboratoire franco-japonais sur la fusion magnétique (FJ-MFL) par le CNRS, et d'un Institut international des Sciences de la Fusion par les universités de Marseille. Signalons enfin la décision toute récente de doter l'expérience Tore Supra du CEA à 
Cadarache d'un divertor en tungstène (projet WEST) pouvant fonctionner pendant des pulses longs dans les conditions de charge thermique d'ITER. La vocation de WEST est de devenir une plateforme expérimentale ouverte aux équipes françaises et internationales.

Dans le secteur de l'enseignement supérieur, on notera aussi la création en 2006 d'un master national sur les Sciences de la fusion, ainsi que la participation de l'Université Henri Poincaré de Nancy au master Erasmus Mundus Fusion EP (Engineering Physics). À partir de 2013, l'ensemble de ces étudiants (environ 40 personnes) sera regroupé à Cadarache pendant plusieurs semaines pour un premier contact avec les recherches en fusion magnétique.

Les technologies avancées d'ITER ont toutes été démontrées sur des prototypes, certains à l'échelle 1. C'est le cas, en particulier, du grand solénoïde central en $\mathrm{Nb}_{3} \mathrm{Sn}$ qui a battu largement tous les records pour un solénoïde pulsé jusqu'à un champ magnétique dépassant $11 \mathrm{~T}$ sur le conducteur. Les défis techniques concernent donc essentiellement l'intégration de ces technologies, avec de multiples interfaces de composants en provenance des quatre coins du monde. On attirera l'attention sur le fait que les composants intérieurs de la chambre à vide devront être activement refroidis, ce qui impose des exigences particulières pour leur réalisation.

La fluence neutronique sur les composants d'ITER ne pose pas de problème de matériaux particulier. Ce ne sera pas le cas pour un futur réacteur, aspect qui fait l'objet de la contribution de J.L. Boutard (à paraître dans le n³3 de Reflets de la physique).

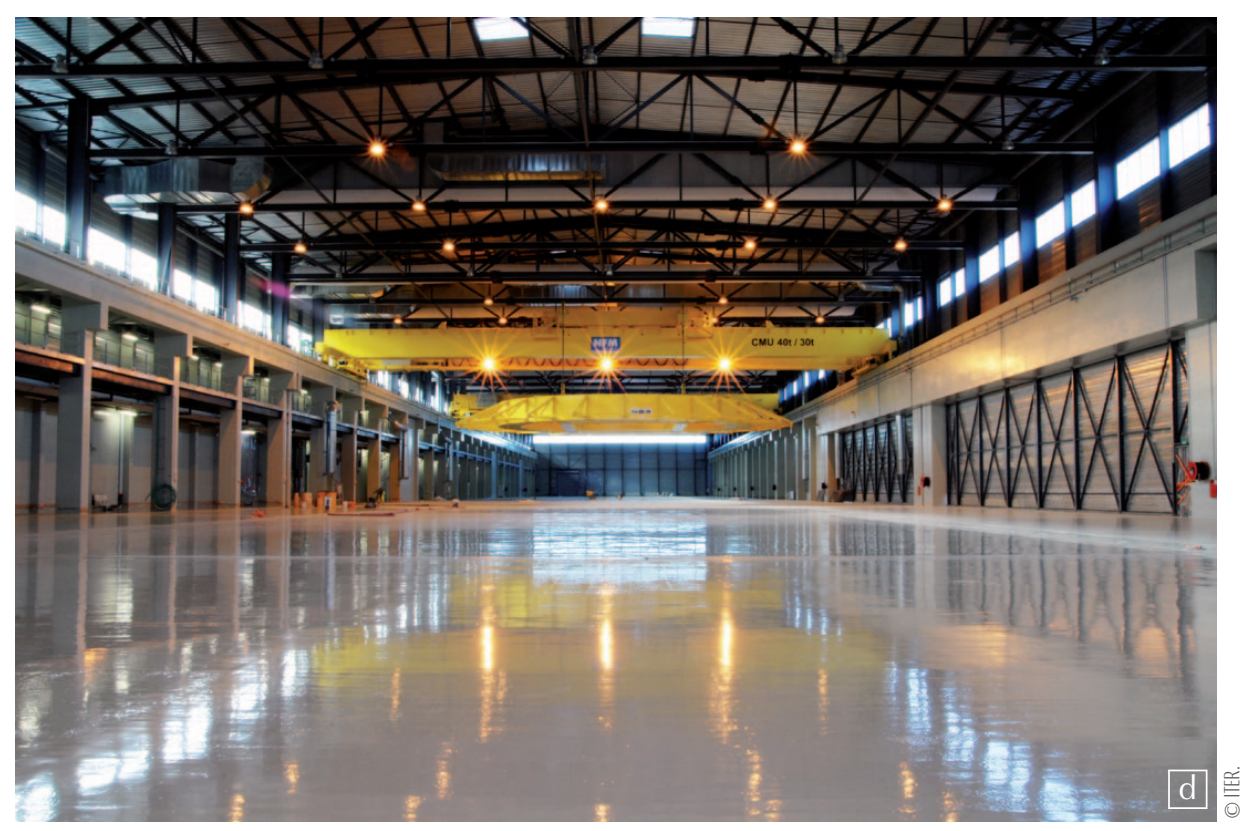

\section{Références}

1 M. Decroisette, « La fusion thermonucléaire par laser », Reflets de la physique n²1 (2010), pp. 35-38.

2- J. Jacquinot, "Fifty years in fusion and the way forward", Nucl. Fusion 50 (2010) 014001.

3• M. Keilhacker et al., "High fusion performance from deuterium-tritium plasmas in JET", Nucl. Fusion 39 (1999) 209.

4- J. Jacquinot et al., "Recent developments in steadystate physics and technology of tokamaks in Cadarache", Nucl. Fusion 43 (2003) 1583.

$5 \bullet G$. Laval et al., La Fusion Nucléaire : de la recherche fondamentale à la production d'énergie?., Rapport scientifique et technique n²6 de l'Académie des sciences, EDP Sciences (2007)

6•X. Garbet et al., "Physics of transport in tokamaks", Plasma Phys. Control. Fusion 46 (2004) B557.

7• "Burning Plasma: Bringing a Star to Earth", The National Academies Press, Washington, USA (2004) http://fire.pppl.gov/nrc_bpac_draft_prepub.ppf

\section{Ouvrages généraux}

- J.L. Bobin, La fusion thermonucléaire contrôlée. EDP Sciences (2011).

- A. Benuzzi-Mounaix, La fusion nucléaire, Belin (2008).

- G. Laval, L'énergie bleue, Odile Jacob (2007).

- R. Arnoux et J. Jacquinot, ITER, le chemin des étoiles ? Edisud (2006).

- J. Weisse, La fusion nucléaire, Que sais-je ? Puf (2003

- P.H. Rebut, L'énergie des étoiles, la fusion nucléaire contrôlée, Odile Jacob (1999)

Le projet ITER se heurte à l'opposition de la mouvance écologiste, mais aussi de certains milieux scientifiques (a). II a fait l'objet d'un débat public en 2006 (b).

Ces controverses sont abordées avec une grande objectivité dans l'ouvrage de A. Benuzzi-Mounaix, cité ci-dessus (pp. 73-75).

(a) G. Charpak, J. Treiner, S. Balibar, dans Libération du 10 août 2010.

(b) http://cpdp.debatpublic.fr/cpdp-iter/

\section{Glossaire}

JET : Joint European Tokamak, exploité à Culham (UK).

Rayon de Larmor : rayon de la trajectoire circulaire d'une particule chargée soumise à un champ magnétique constant B. En mécanique classique, il vaut $\mathrm{mv} / \mathrm{ZeB}$, où $\mathrm{m}, \mathrm{v}$ et Ze sont respectivement la masse, la vitesse et la charge de la particule.

Stellarator : dispositif de fusion nucléaire par confinement magnétique, différent des tokamaks, et dans lequel le confinement du plasma est réalisé par un champ magnétique hélicö̈dal créé par un arrangement complexe de bobines extérieures au tore. Cette configuration est conçue pour fonctionner en régime continu. Plusieurs machines sont en développement, aux USA, au Japon et en Allemagne. La physique du confinement n'est pas très différente de celle des tokamaks.

Vitesse d'Alfven : vitesse de propagation d'un certain type d'ondes de champ magnétique de basse fréquence dans un plasma.

Temps de confinement de l'énergie $\left(\tau_{\mathrm{F}}\right)$ : temps caractéristique de diffusion de l'énergie vers le bord du plasma ; en régime stationnaire, il vaut W/P où W est l'énergie contenue dans le plasma et P la puissance de chauffage (fusion + puissance de chauffage auxiliaire). 